\title{
KamLAND Data and the Solution to the Solar Neutrino Problem
}

\author{
P. C. de Holanda ${ }^{1}$ and A. Yu. Smirnov ${ }^{2,3}$ \\ ${ }^{1}$ Instituto de Física Gleb Wataghin - UNICAMP, 13083-970 Campinas SP, Brazil \\ ${ }^{2}$ The Abdus Salam International Centre for Theoretical Physics, I-34100 Trieste, Italy \\ ${ }^{3}$ Institute for Nuclear Research of Russian Academy of Sciences, Moscow 117312, Russia
}

Received on 17 November, 2003

\begin{abstract}
The first KamLAND results are in a very good agreement with the predictions made on the basis of the solar neutrino data and the LMA realization of the MSW mechanism. We perform a combined analysis of the KamLAND (rate, spectrum) and the solar neutrino data with a free boron neutrino flux $f_{B}$. The best fit values of neutrino parameters are $\Delta m^{2}=7.1 \cdot 10^{-5} \mathrm{eV}^{2}, \tan ^{2} \theta=0.40$ and $f_{B}=1.04$ with the $1 \sigma$ intervals: $\Delta m^{2}=(6.4-8.4) \cdot 10^{-5} \mathrm{eV}^{2}, \tan ^{2} \theta=0.33-0.48$. We find the $3 \sigma$ upper bounds: $\Delta m^{2}<1.7 \cdot 10^{-4}$ $\mathrm{eV}^{2}$ and $\tan ^{2} \theta<0.64$, and the lower bound $\Delta m^{2}>4.8 \cdot 10^{-5} \mathrm{eV}^{2}$. In the best fit point we predict for SNO: $\mathrm{CC} / \mathrm{NC}=0.32_{-0.07}^{+0.08}$ and $A_{D N}^{S N O}=3.0 \pm 0.8 \%$ (68\% C.L.), and $A_{D N}^{S N O}<6 \%$ at the $3 \sigma$ level. Further improvements in the determination of the oscillation parameters are discussed and implications of the solar neutrino and KamLAND results are considered.
\end{abstract}

\section{Introduction}

The first KamLAND results [1] are the last (or almost last) step in resolution of the long-standing solar neutrino problem [2]. In fact, KamLAND excludes also all nonoscillation solutions based on neutrino spin-flip in the magnetic fields of the Sun, on the non-standard neutrino interactions, etc.. More precisely, KamLAND excludes them as the dominant mechanisms of the solar neutrino conversion.

By the time of the KamLAND announcement, the solar neutrino data [3-10] have definitely selected LMA as the most favorable solution based on neutrino mass and mixing [11-13]. After the publication of KamLAND results, SNO experiment published their results on the salt phase detection [14], confirming LMA predictions and restricting even more the allowed region for neutrino parameters. The best fit point from the free boron neutrino flux fit [15] is

$$
\begin{aligned}
\Delta m^{2} & =6.31 \cdot 10^{-5} \mathrm{eV}^{2} \\
\tan ^{2} \theta & =0.39 \\
f_{B} & =1.06,
\end{aligned}
$$

where $f_{B} \equiv F_{B} / F_{B}^{S S M}$ is the boron neutrino flux in the units of the Standard Solar Model predicted flux [16].

On basis of the solar neutrino results (and the assumption of the CPT invariance) predictions for the KamLAND experiment have been calculated. A significant suppression of the signal was expected in the case of the LMA solution. The predicted ratio of the numbers of events with the visible (prompt) energies, $E_{p}$, above $2.6 \mathrm{MeV}$ with and without oscillations equals [13]:

$$
R_{K L}^{L M A}=0.65_{-0.38}^{+0.08} \quad(3 \sigma) .
$$

For other solutions of the solar neutrino problem one expected $R_{K L}=0.9-1$, where the deviation from 1 can be due to the effect of nonzero 1-3 mixing.

In the best fit point (1) the predicted spectrum has (i) a peak at $E_{p} \approx(3.0-3.6) \mathrm{MeV}$, (ii) a suppression of the number of events near the threshold energy $E_{p} \approx 2.6 \mathrm{MeV}$ and (iii) a significant suppression of the signal (with respect to the no-oscillation case) at the high energies: $E_{p}>(4-5)$ $\mathrm{MeV}$ [13]. No distortion of the spectrum is expected for the other solutions.

The first KamLAND results, both the total number of events and spectrum shape [1], are in a very good agreement with predictions:

$$
R_{K L}^{\exp }=0.611 \pm 0.094
$$

The spectral data (although not yet precise) reproduce well the features described above. As a result, the allowed "island" in the $\Delta m^{2}-\sin ^{2} 2 \theta$ plane with the best fit KamLAND point covers the best fit point from the solar neutrino analysis [1].

This work is based in our recent paper $[17,15]$ where the KamLAND data were analysed, as well as the combination of this analysis with solar neutrinos data.

\section{KamLAND}

The KamLAND (Kamioka Liquid scintillator Anti-Neutrino Detector) experiment detects anti-neutrinos created in $\sim 26$ nuclear reactors situated around the detector site, tipically at distances between 80 and $200 \mathrm{Km}$. It consists of a very large volume scintilator detector, that can detect the anti-neutrinos from the inverse beta-decay reaction:

$$
\overline{\nu_{e}}+p \rightarrow e^{+}+n
$$


In a given energy bin $a(a=1, \ldots .13)$ the signal at KamLAND is determined by

$$
N_{a}=A \sum_{i} \int_{E_{a}}^{E_{a}+\Delta E} d E_{p} \int d E_{p}^{\prime} P_{i} F_{i} \sigma f\left(E_{p}, E_{p}^{\prime}\right),
$$

where $\Delta E=0.425 \mathrm{MeV}$,

$$
P_{i}=\left(1-\sin ^{2} 2 \theta_{12} \sin ^{2} \frac{\Delta m_{12}^{2} L_{i}}{4 E}\right)
$$

is the vacuum oscillation survival probability for $i$ reactor situated at the distance $L_{i}$ from KamLAND, $F_{i}$ is the flux from $i$ reactor, $\sigma$ is the cross-section of $\bar{\nu} p \rightarrow e^{+} n$ reaction, $E_{p}$ is the observed prompt energy, $E_{p}^{\prime}$ is the true prompt energy, $f\left(E_{p}, E_{p}^{\prime}\right)$ is the energy resolution function, $A$ is the factor which takes into account the fiducial volume, the time of observation, etc.. We sum over all reactors contributing appreciable to the flux at KamLAND.

The suppression factor of the total number of (reactor neutrino) events above certain threshold is defined as:

$$
R_{K L}\left(\Delta m^{2}, \tan ^{2} \theta\right) \equiv \frac{N\left(\Delta m^{2}, \tan ^{2} \theta\right)}{N_{0}}
$$

where $N=\sum_{a} N_{a}, N_{a}$ is given in (4) and $N_{0}$ is the total number of events in the absence of oscillations (following KamLAND we will call $N$ and $N_{0}$ the rates).

1). KamLAND spectrum. The KamLAND data are analyzed through a Poisson statistics, using the following $\chi^{2}$ :

$$
\chi^{2}=\sum_{i=1,13} 2\left[N_{i}^{t h}-N_{i}^{o b s}+N_{i}^{o b s} \ln \left(\frac{N_{i}^{o b s}}{N_{i}^{t h}}\right)\right]
$$

where the $\ln$ term is absent in bins with no events (5 last bins).

We find that for $E_{p} \geq 2.6 \mathrm{MeV}$ the minimum of $\chi_{\text {spec }}^{2}$ is achieved for

$$
\Delta m^{2}=7.2 \cdot 10^{-5} \mathrm{eV}^{2}, \quad \tan ^{2} \theta=0.52,
$$

and in this point $\chi^{2} /$ d.o.f. $=5.91 / 11$. Notice that in contrast with the KamLAND result [1] our best fit mixing deviates from the maximal mixing.

We present in Fig. 1 the contours of constant confidence level with respect to the best fit point (7) in the $\left(\Delta m^{2}-\tan ^{2} \theta\right)$ plane using relation: $\chi^{2}=\chi_{\min }^{2}+\Delta \chi^{2}$, where $\Delta \chi^{2}=1,3.84$ and 6.63 for $1 \sigma, 95 \%$ and $99 \%$ C.L. correspondingly.

The contours manifest an oscillatory pattern in $\Delta m^{2}$ in spite of a strong averaging effect which originates from large spread in distances from different reactors. The pattern can be described in terms of oscillations with certain effective distance, $L_{e f f}$, and effective oscillation phase $\phi_{e f f}$ :

$$
\phi_{e f f}=\frac{\Delta m_{12}^{2} L_{e f f}}{4 E}, \quad L_{e f f} \approx 165 \mathrm{~km} .
$$

$L_{\text {eff }}$ corresponds to the distance between KamLAND and the closest set of reactors which provides the large fraction of the antineutrino flux.

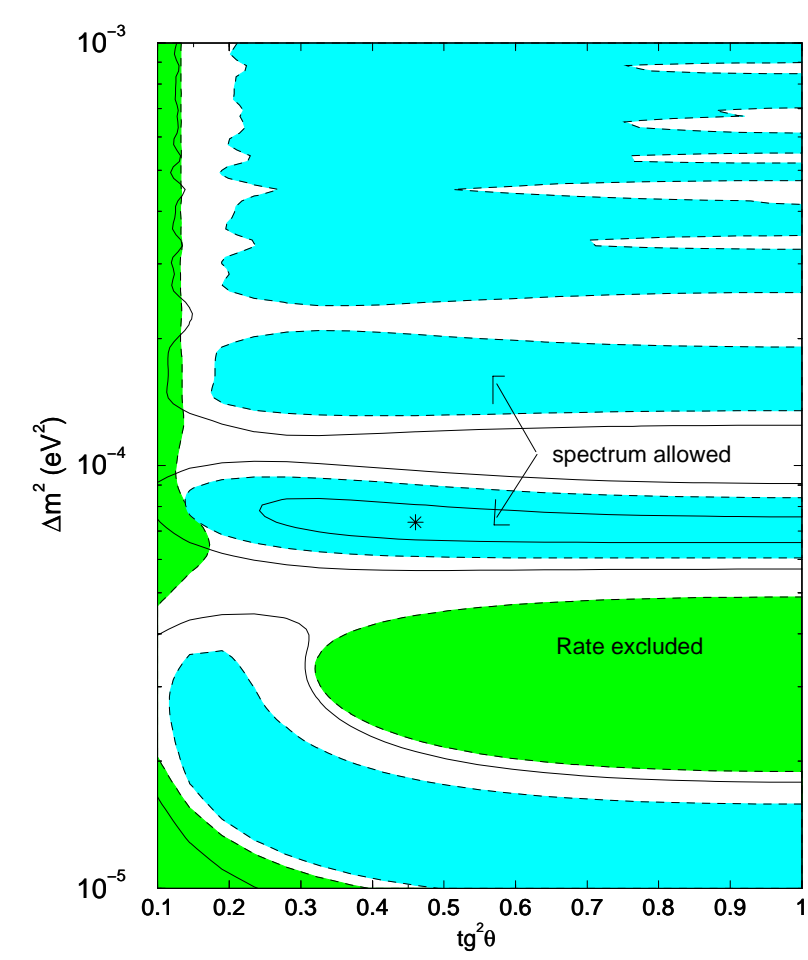

Figure 1. The KamLAND spectrum analysis for $E_{p}>2.6 \mathrm{MeV}$. Shown are the allowed regions of oscillation parameters at $68 \%$ (inner solid lines), 95\% (grey) and 99\% C.L. (outer solid lines). The best fit point is indicated by star. Also shown are the regions excluded by the rate analysis at $95 \%$ C.L. (dark).

Let us consider the $95 \%$ allowed regions.

(i) The lowest "island" allowed by KamLAND with $\Delta m^{2}<2 \cdot 10^{-5} \mathrm{eV}^{2}$ corresponds to the oscillation phase $\phi_{\text {eff }}<\pi / 2$. This region is excluded by the absence of significant day-night asymmetry of the Super-Kamiokande signal. In this domain, the predicted asymmetry at SNO, $A_{D N}^{S N O}>17 \%$, is still consistent with data.

(ii) The second allowed region, $\Delta m^{2}=(5-10) \cdot 10^{-5}$ $\mathrm{eV}^{2}$, corresponds to the first oscillation maximum, $\phi_{\text {eff }} \sim$ $\pi$ (maximum of the survival probability). It contains the best fit point.

(iii) The third island is at $\Delta m^{2}=(13-23) \cdot 10^{-5} \mathrm{eV}^{2}$ : it corresponds to the oscillation maximum (second maximum of the survival probability) with $\phi_{\text {eff }} \sim 2 \pi$.

There is a continuum of the allowed regions above $\Delta m^{2} \sim 3 \cdot 10^{-4} \mathrm{eV}^{2}$. The third region merges with the continuum at $99 \% \mathrm{CL}$.

At the $1 \sigma$ level the second island is the only allowed region.

2) KamLAND rate. In Fig. 1 we show the regions excluded by the KamLAND rate at $95 \%$ C.L.. The borders of these regions coincide with contours of constant $R_{K L}^{\max }=0.80$ and $R_{K L}^{\min }=0.42$ obtained in [13]). The exclusion region at $\Delta m^{2} \sim(2-5) \cdot 10^{-5} \mathrm{eV}^{2}$ corresponds to the first oscillation minimum (minimum of the survival probability) at KamLAND $\left(\phi_{\text {eff }} \sim \pi / 2\right)$. Here the suppression of the 
signal is too strong. Another region of significant suppression (second oscillation minimum with $\phi_{\text {eff }}=3 \pi / 2$ ) is at $\Delta m^{2} \sim(9-12) \cdot 10^{-5} \mathrm{eV}^{2}$.

3). KamLAND spectrum and rate. Following procedure in [1] we have performed also combined analysis of spectrum and rate introducing the free normalization parameter of the spectrum, $R_{K L}$, and defining the $\chi^{2}$ as

$$
\chi_{\text {spec }, R}^{2}=\chi_{\text {spec }}^{2}+\chi_{R}^{2},
$$

where

$$
\chi_{R}^{2}=\left(\frac{R_{K L}-0.611}{0.094}\right)^{2} .
$$

We find results which are very close to those from our spectrum analysis. In particular, the best fit value of mixing is $\tan ^{2} \theta=0.48$ and $\Delta m^{2}=7.31 \cdot 10^{-5} \mathrm{eV}^{2}$.

As it follows from our consideration here, the values of oscillation parameters extracted from the KamLAND data, $\left(\Delta m^{2}, \tan ^{2} \theta\right)_{K L}$, are in a very good agreement with the values from independent solar neutrino analysis $\left(\Delta m^{2}, \tan ^{2} \theta\right)_{\text {sun }}$. For the best fit points (1), (7) we conclude that within $1 \sigma$ (see Fig. 1 )

$$
\left(\Delta m^{2}, \tan ^{2} \theta\right)_{K L}=\left(\Delta m^{2}, \tan ^{2} \theta\right)_{\text {sun }} .
$$

At the same time, the data do not exclude that the solar and KamLAND parameters are different, and moreover, the difference still can be large. For instance, $\left(\Delta m^{2}, \tan ^{2} \theta\right)_{K L}$ can coincide with the present best fit point or be in the high $\Delta m^{2}$ island, whereas $\left(\Delta m^{2}, \tan ^{2} \theta\right)_{\text {sun }}$ can be at lower $\Delta m^{2}$.

\section{Solar Neutrinos}

We use the same data set and the same procedure of analysis as in our previous publication [15]. Here the main ingredients of the analysis are summarized.

The data sample consists of

- 3 total rates: (i) the $A r$-production rate, $Q_{A r}$, from Homestake [3], (ii) the $G e-$ production rate, $Q_{G e}$ from SAGE [5] and (iii) the combined $G e$-production rate from GALLEX and GNO [6];

- 44 data points from the zenith-spectra measured by SuperKamiokande during 1496 days of operation [7];

- 34 day-night spectral points from SNO $[9,10]$.

- CC, NC and ES rates from SNO salt-phase [14]

Altogether the solar neutrino experiments provide us with 84 data points. The SNO salt phase data were issued after the publication of the first KamLAND data analyzed here. Nevertheless we included it in our solar scenario since they have an important role in discriminating the $\Delta m^{2}$ degeneracy that arises from KamLAND analysis.

All the solar neutrino fluxes, but the boron neutrino flux, are taken according to SSM BP2000 [16]. The boron neutrino flux is treated as a free parameter. For the $h e p$-neutrino flux we take fixed value $F_{h e p}=9.3 \times 10^{3}$ $\mathrm{cm}^{-2} \mathrm{~s}^{-1}[16,18]$.
Thus, in our analysis of the solar neutrino data as well as in the combined analysis of the solar and KamLAND results we have three fit parameters: $\Delta m^{2}, \tan ^{2} \theta$ and $f_{B}$.

We define the contribution of the solar neutrino data to $\chi^{2}$ as

$$
\chi_{\text {sun }}^{2}=\chi_{\text {rate }}^{2}+\chi_{S K}^{2}+\chi_{S N O}^{2}+\chi_{S N O-I I}^{2},
$$

where $\chi_{\text {rate }}^{2}, \chi_{S K}^{2}, \chi_{S N O}^{2}$ and $\chi_{S N O-I I}^{2}$ are the contributions from the total rates, the Super-Kamiokande zenith spectra, the SNO day and night spectra and the SNO salt phase correspondingly. The main result of analysis performed in [15] is given here in Eq. (1).

\section{Solar neutrinos and KamLAND}

We have performed two different combined fits of the data from the solar neutrino experiments and KamLAND.

1) KamLAND rate and solar neutrino data. There are 84 $($ solar $)+1$ (KamLAND) data points -3 free parameters $=$ 82 d.o.f.. We define the global $\chi^{2}$ for this case as

$$
\chi_{\text {sun }, R}^{2}=\chi_{\text {sun }}^{2}+\chi_{R}^{2}
$$

where $\chi_{\text {sun }}^{2}$ and $\chi_{R}^{2}$ are given in (12) and (10). The minimum $\chi_{\text {sun }, R}^{2}(\min ) /$ d.o.f. $=67.2 / 82$ corresponds to the C.L. $=88 \%$. It appears at

$$
\begin{aligned}
\Delta m^{2} & =5.58 \cdot 10^{-5} \mathrm{eV}^{2} \\
\tan ^{2} \theta & =0.39 \\
f_{B} & =1.08 .
\end{aligned}
$$

This point practically coincides with what we have obtained from the solar neutrino analysis only.

We construct the contours of constant confidence level in the $\left(\Delta m^{2}-\tan ^{2} \theta\right)$ plot using the following procedure. We perform minimization of $\chi_{\text {sun, } R}^{2}$ with respect to $f_{B}$ for each point of the oscillation plane, thus getting $\chi_{\text {sun }, R}^{2}\left(\Delta m^{2}, \tan ^{2} \theta\right)$. Then the contours are defined by the condition $\chi_{\text {sun }, R}^{2}\left(\Delta m^{2}, \tan ^{2} \theta\right)=\chi_{\text {sun }, R}^{2}(\min )+\Delta \chi^{2}$, where $\Delta \chi^{2}=2.3,4.61,5.99,9.21$ and 11.83 are taken for $1 \sigma, 90 \%, 95 \%$ and $99 \%$ C.L. and $3 \sigma$. The results are shown in Fig. 2. 


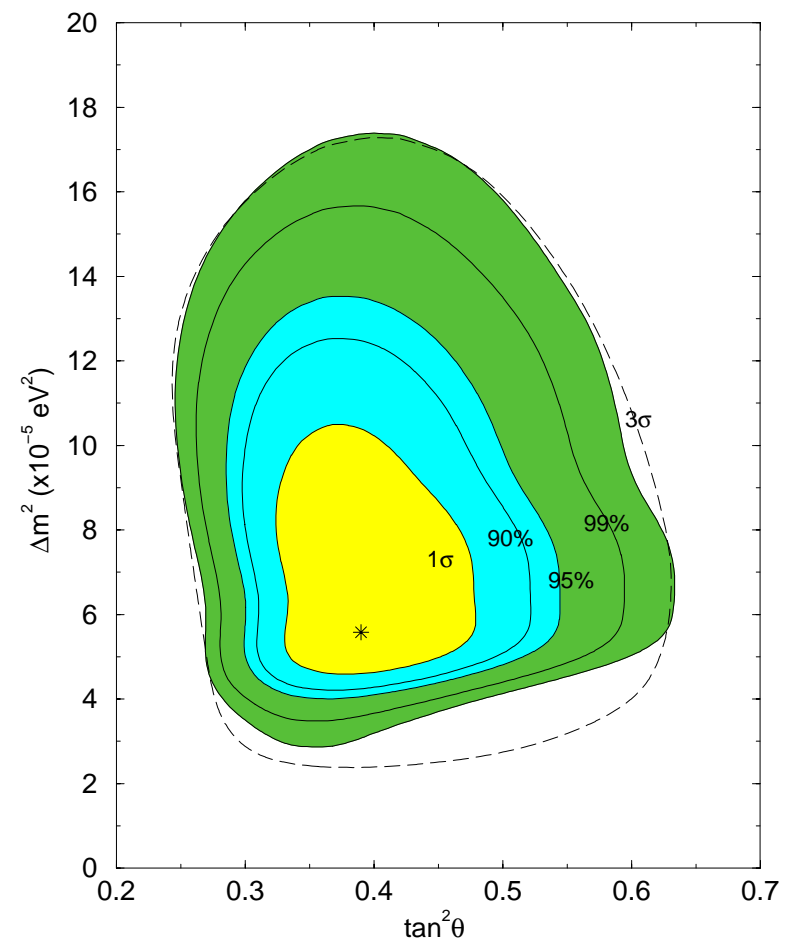

Figure 2. The allowed regions in $\tan ^{2} \theta-\Delta m^{2}$ plane from a combined analysis of the solar neutrino data and the KamLAND rate, at $1 \sigma, 90 \%, 95 \%, 99 \%$ and $3 \sigma$ C.L.. The best fit point is marked by star. Also shown in dashed lines the allowed region at 99\% C.L. with only solar data.

According to the figure, the main impact of the KamLAND rate is strengthening of bound on the allowed region from below due to strong suppression of the KamLAND rate at $\Delta m^{2}=(2-5) \cdot 10^{-5} \mathrm{eV}^{2}$ (see Fig. 1) - region of the first oscillation minimum in KamLAND. The lines of constant confidence level are shifted to larger $\Delta m^{2}$. The KamLAND rate leads to a distortion (shift to smaller mixings) of contours at $\Delta m^{2} \sim 10^{-4} \mathrm{eV}^{2}$ where the second oscillation minimum at KamLAND is situated. The upper part of the allowed region is modified rather weakly.

2). KamLAND spectrum and solar neutrino data. We calculate

$$
\chi_{\text {global }}^{2}=\chi_{\text {sun }}^{2}+\chi_{\text {spec }}^{2},
$$

where $\chi_{\text {spec }}^{2}$ has been defined in (2). In this case we have 84 (solar) + 13 (KamLAND) data points - 3 free parameters $=94$ d.o.f.. The absolute minimum, $\chi_{\text {global }}^{2}($ min $)=73.4$ (which corresponds to a very high confidence level: $94 \%$ ), is at

$$
\begin{aligned}
\Delta m^{2} & =7.13 \cdot 10^{-5} \mathrm{eV}^{2} \\
\tan ^{2} \theta & =0.40 \\
f_{B} & =1.038 .
\end{aligned}
$$

The best fit value of $\Delta m^{2}$ is slightly higher than that from the solar data analysis. The solar neutrino data have higher sensitivity to mixing, whereas the KamLAND is more sensitive to $\Delta m^{2}$, as a result, in (16) the value of $\Delta m^{2}$ is close to the one determined from the KamLAND data only (7),

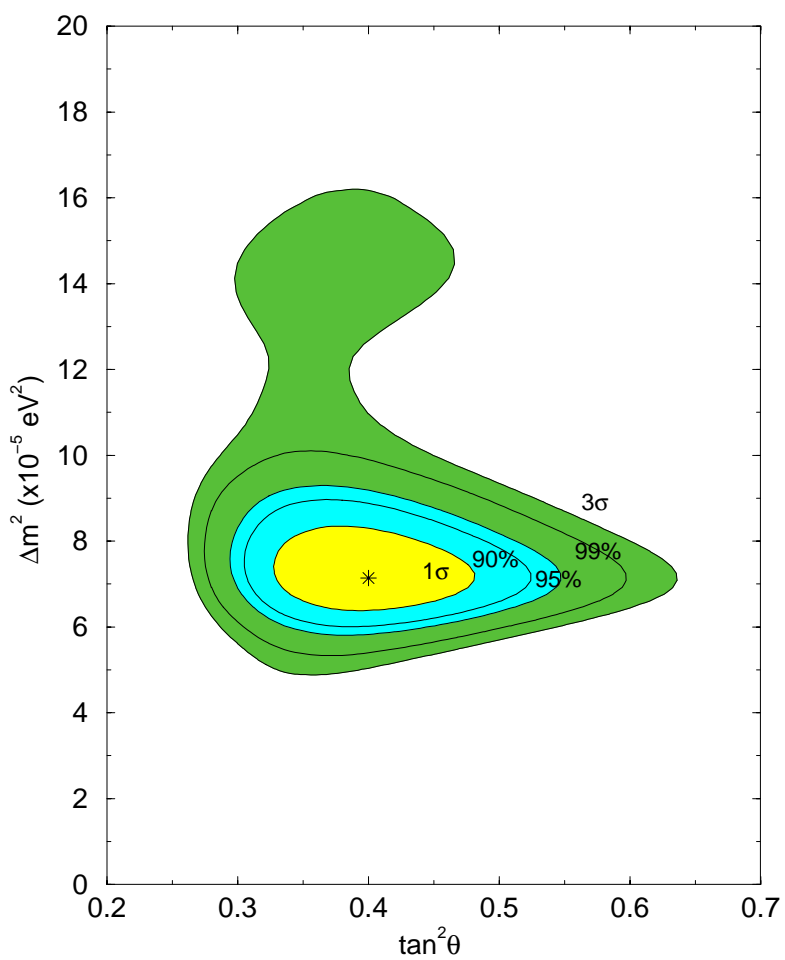

Figure 3. The allowed regions in $\tan ^{2} \theta-\Delta m^{2}$ plane, from a combined analysis of the solar neutrino data and the KamLAND spectrum at $1 \sigma, 90 \%, 95 \%, 99 \%$ and $3 \sigma$ C.L.. The best fit point is marked by star.

whereas $\tan ^{2} \theta$ almost coincides with mixing determined from the solar neutrino results (1).

We construct the contours of constant confidence levels in the oscillation plane, similarly to what we did for the fit of the solar data and the KamLAND rate (Fig. 3).

As compared with the solar data analysis, KamLAND practically has not changed the upper bound on mixing or $\Delta m^{2}$. At the $3 \sigma$ level we get:

$\Delta m^{2}>4.8 \cdot 10^{-5} \mathrm{eV}^{2}, \quad \tan ^{2} \theta<0.64, \quad 99.73 \%$ C.L.

The spectral data disintegrate the LMA region. At the $3 \sigma$ level only a small spot is left in the range $\Delta m^{2}>1.2 \cdot 10^{-4}$ $\mathrm{eV}^{2}$.

The region "splits" into two regions, above and below $\Delta m^{2}>1.2 \cdot 10^{-4} \mathrm{eV}^{2}$, which we will refer to as the lower (1-) and higher (h-) LMA regions. (Existence of these two regions can be seen already from an overlap of the solar and KamLAND allowed regions in [1]).

The shrink in allowed region around $\Delta m^{2} \sim 1.2$. $10-4 \mathrm{eV}^{2}$ corresponds to the second oscillation minimum $\left(\phi_{\text {eff }} \sim 3 \pi / 2\right)$ at $E_{p} \sim(3-4) M e V$ which contradicts the spectral data.

Features of spectrum distortion. In the Fig. 4 we show the prompt energy spectra of events for the best fit points in the 1- and h-regions, $N_{l}\left(E_{p}\right)$ and $N_{h}\left(E_{p}\right)$. The spectra can be 


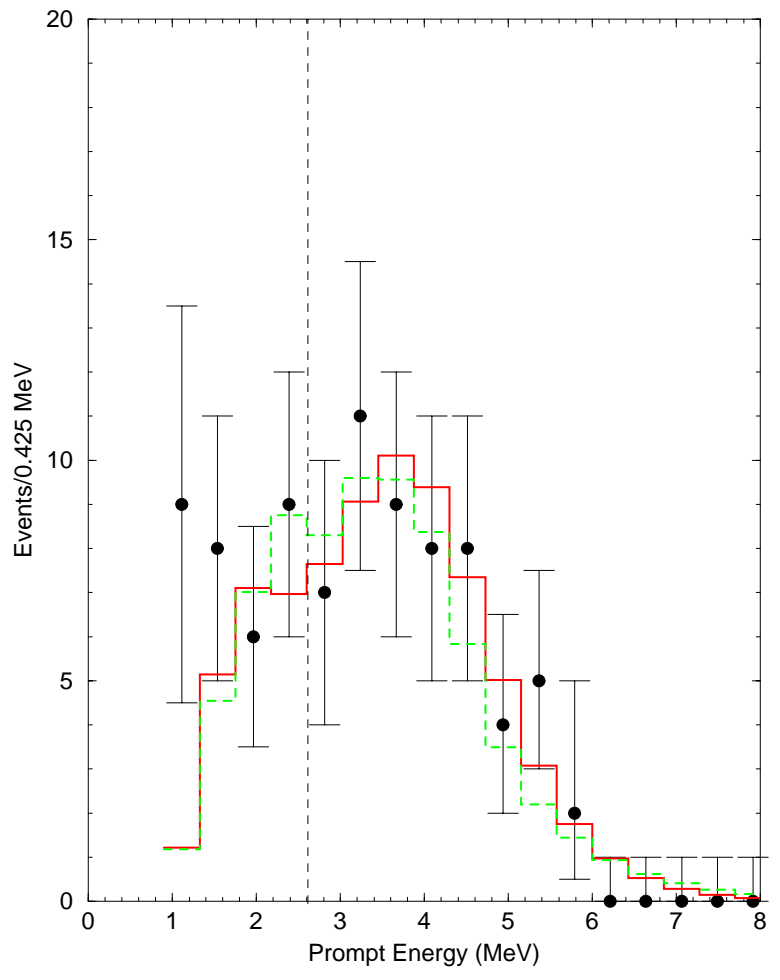

Figure 4. The expected prompt energy spectra for the best fit points from the l-region (solid histogram) and h-region (dashed histogram). Also shown are the KamLAND experimental points.

well understood in terms of the effective oscillation phase $\phi_{e f f}$ :

$$
N\left(E_{p}\right) \sim N_{0}\left(E_{p}\right)\left[1+D\left(E_{p}\right) \sin ^{2} \phi_{e f f}\right],
$$

where $D\left(E_{p}\right)$ is the averaging factor.

In the best fit point of l-region (16), the peak at $E_{p} \approx 3.6$ $\mathrm{MeV}$ corresponds to the oscillation maximum $\phi_{\text {eff }}=\pi$ (exact position of maximum of the survival probability is at $E_{p}=4.3 \mathrm{MeV}$ ). The closest oscillation minimum (phase $\phi_{\text {eff }}=3 \pi / 2$ ) is at $E_{p} \approx 2.4 \mathrm{MeV}$ and the next maximum $\left(\phi_{\text {eff }}=2 \pi\right)$ is at $E_{p} \approx 1.8 \mathrm{MeV}$. Due to strong averaging effect the structures below (in $E_{p}$ ) the main maximum are not profound and look more like a shoulder below the peak. The first oscillation minimum is at $E_{p}=7.2 \mathrm{MeV}$.

The measured spectrum, indeed, gives a hint of existence of the low energy shoulder. Evidently with the present data it is impossible to disentangle the l- and h-spectra. Substantial decrease of errors is needed. Also decrease of the energy threshold will help. According to Fig. $4, N_{l}\left(E_{p}\right)>N_{h}\left(E_{p}\right)$ at $E_{p}>3.5 \mathrm{MeV}$, and $N_{l}\left(E_{p}\right)<N_{h}\left(E_{p}\right)$ at lower energies, especially in the interval $E_{p}=(2.0-2.5) \mathrm{MeV}$. Therefore for the low threshold the difference between $N_{l}\left(E_{p}\right)$ and $N_{h}\left(E_{p}\right)$ can not be eliminated by normalization (mixing angle).

For convenience, the results of different fits are summarized in the Table 1. It shows high stability of the extracted parameters with respect to a type of analysis.

\section{$5 \quad$ Next step}

The key problems left after the first KamLAND results are

- more precise determination of the neutrino parameters: in particular, (i) precise determination of the deviation of 12-mixing from maximal mixing, (ii) strengthening of the upper bound on $\Delta m^{2}$ (iii) discrimination between the two existing regions;

- searches for effects beyond the single $\Delta m^{2}$ and single mixing approximation;

- searches for differences of the neutrino oscillation parameters determined from KamLAND and from the solar neutrino experiments.

Notice that precise knowledge of the parameters is crucial not only for the neutrinoless double beta decay searches, long baseline experiments, studies of the atmospheric and supernova neutrinos, etc., but also for understanding of physics of the solar neutrino conversion. In the region of the best fit point a dominating process (at least for $E>(0.5-1)$ $\mathrm{MeV}$ ) is the adiabatic neutrino conversion (MSW), whereas in the high $\Delta m^{2}$ region allowed by KamLAND at the $2 \sigma$ level, the effect is reduced to the averaged vacuum oscillations (a la Gribov-Pontecorvo) [19] with small matter corrections.

In this connection, we will discuss two questions.

How small $\Delta m^{2}$ can be? This is especially important question, e.g., for measurements of the earth regeneration effect. In the l-region we get

$$
\Delta m^{2}>4.8 \cdot 10^{-5} \mathrm{eV}^{2},
$$

and it is difficult to expect that lower values will be allowed.

The bound (19) appears as an interplay of both the KamLAND rate and the shape. As it follows from the Fig. 2, the KamLAND rate strengthens the lower bounds: $\Delta m^{2} \geq(2.7,3.7,4.5) \times 10^{-5} \mathrm{eV}^{2}$, at the $1 \sigma, 2 \sigma, 3 \sigma$ correspondingly which should be compared with $\Delta m^{2} \geq$ $(2.2,2.9,3.8) \times 10^{-5} \mathrm{eV}^{2}$ from the solar analysis only. Adding the spectral data results in the bounds $\Delta m^{2} \geq$ 4.8, 5.6, $6.2 \times 10^{-5} \mathrm{eV}^{2}$

With decrease of $\Delta m^{2}$ the oscillatory pattern of the spectrum shifts to lower energies. For $\Delta m^{2}=5 \cdot 10^{-5} \mathrm{eV}^{2}$ the maximum of spectrum is at $E_{p}=2.7 \mathrm{MeV}$ and the oscillation suppression increases with energy [13]. The oscillation minimum is at $E_{p} \approx 5 \mathrm{MeV}$. If $R_{K L}(2.7 \mathrm{MeV})=0.81$, then $R_{K L}(4.0 \mathrm{MeV})=0.47$. The KamLAND spectrum does not show such a fast decrease.

One can characterize the spectrum distortion by a relative suppression of signal at the high (say, above 4.3 MeV) and at the low (below $4.3 \mathrm{MeV}$ ) energies The energy interval (2.6 - 4.3) MeV contains KamLAND energy 4 bins. Introducing the suppression factors $R_{K L}(<4.3 \mathrm{MeV})$ and $R_{K L}(>4.3 \mathrm{MeV})$ we can define the ratio

$$
k=\frac{1-R_{K L}(>4.3 \mathrm{MeV})}{1-R_{K L}(<4.3 \mathrm{MeV})} .
$$


$k$ which we will call the shape parameter does not depend on the mixing angle and normalization of spectrum. It increases with increase of the oscillation suppression at high energies.

Using the KamLAND data we get the experimental value

$$
k^{e x p}=0.84_{-0.35}^{+.42}, \quad 1 \sigma \text {. }
$$

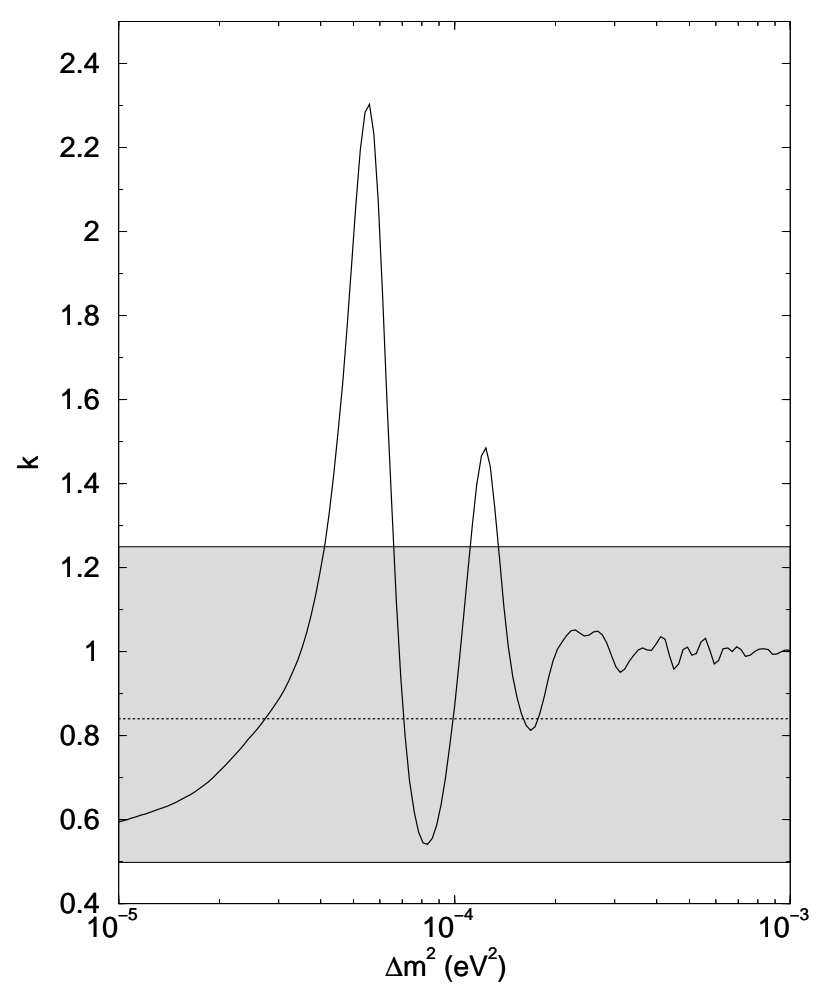

Figure 5. The dependence of the shape parameter $k$ on $\Delta m^{2}$. Shown are the central experimental value (dotted line) and the $1 \sigma$ experimental band (shadowed).

In Fig. 5 we present the dependence of the shape parameter $k$ on $\Delta m^{2}$. For the spectrum which corresponds to the best combined fit we find $k=0.70$, whereas for $\Delta m^{2}=5 \cdot 10^{-5} \mathrm{eV}^{2}$ the ratio equals $k=2.0$. For the h-region best point: $k=1.2$.

Notice that in the 1-region below $\Delta m^{2}=8 \cdot 10^{-5} \mathrm{eV}^{2}$, $k$ increases quickly with decrease of $\Delta m^{2}$, reaching a maximal value at $\Delta m^{2}=5.5 \cdot 10^{-5} \mathrm{eV}^{2}$. Below that, the parameter $k$ decreases with $\Delta m^{2}$. In this region, however, the total event rate decreases fast giving the bound on $\Delta m^{2}$. This explains a shift of the allowed (at $3 \sigma$ ) region to smaller mixings with decrease of $\Delta m^{2}$.

Notice also that the central experimental value of $k$ can be reproduced in the both allowed regions (l- and h-). Therefore future precise measurement of spectrum will further sharpen determination of $\Delta m^{2}$ within a given island. To discriminate among the islands one needs to use more elaborated criteria (not just $k$ ) or a complete spectral information.

How large is the large mixing? In contrast to [1] our best fit point is at non-maximal mixing $\left(\sin ^{2} 2 \theta=0.86\right)$ being rather close to the best fit point from the solar neutrino analysis. Similar deviation from maximal mixing has been obtained in our rate+spectrum analysis. Notice that the KamLAND data have weak sensitivity to the mixing (weaker than the solar neutrino data). The allowed regions cover the interval

$$
\tan ^{2} \theta=0.12-1.00 \quad(\theta<\pi / 2), \quad 95 \% \text { C.L.. }
$$

Even at $1 \sigma$ the interval $\tan ^{2} \theta=0.23-1.00(\theta<\pi / 2)$ is allowed. The reason is that KamLAND is essentially the vacuum oscillation experiment (see evaluation of matter effects in KamLAND in [20]), and effects of the vacuum oscillations depend on deviation from maximal mixing which can be characterized by $\epsilon=\left(1 / 2-\sin ^{2} \theta\right)$ quadratically: $P \propto 1-4 \epsilon^{2}$. The matter conversion depends on $\epsilon$ linearly: $P \propto 1-2 \epsilon[21]$.

Notice that maximal mixing is rather strongly disfavored by all measured solar neutrino rates. In the point indicated by KamLAND we predict the charged current to neutral current measurement at SNO, the Argon production rate and the Germanium production rate:

$$
\begin{aligned}
& \frac{\mathrm{CC}}{\mathrm{NC}}=0.51(+3.3 \sigma) \\
& Q_{A r}=3.2 \mathrm{SNU}(+2.5 \sigma) \\
& Q_{G e}=63 \mathrm{SNU}(-1.6 \sigma) .
\end{aligned}
$$

In brackets we show the pulls of the predictions from the best fit experimental values. The new measurements of $\mathrm{CC} / \mathrm{NC}$ ratio at SNO have consideraly strenghened the upper bounds on both mixing and $\Delta m^{2}$. We expect that new KamLAND results could strenghen the bounds on $\Delta m^{2}$.

It is important to "overdetermine" the neutrino parameters measuring all possible observables. This will allow us to make cross-checks of selected solution and to search for inconsistencies which will require extensions of the theoretical context. In this connection let us consider predictions for the forthcoming measurements.

1). Precise measurements of the CC/NC ratio at SNO. In Fig. 6 we show the contours of constant $\mathrm{CC} / \mathrm{NC}$ ratio. We find predictions for the best fit point and the $3 \sigma$ interval:

$$
\frac{\mathrm{CC}}{\mathrm{NC}}=0.33 \pm 0.08, \quad 3 \sigma
$$

Values of $\mathrm{CC} / \mathrm{NC}<0.35$ would exclude the h-region. Since SNO collaboration measured $C C / N C=0.306 \pm 0.035$, the h-LMA region became disfavoured at more then $99 \%$ C.L. 


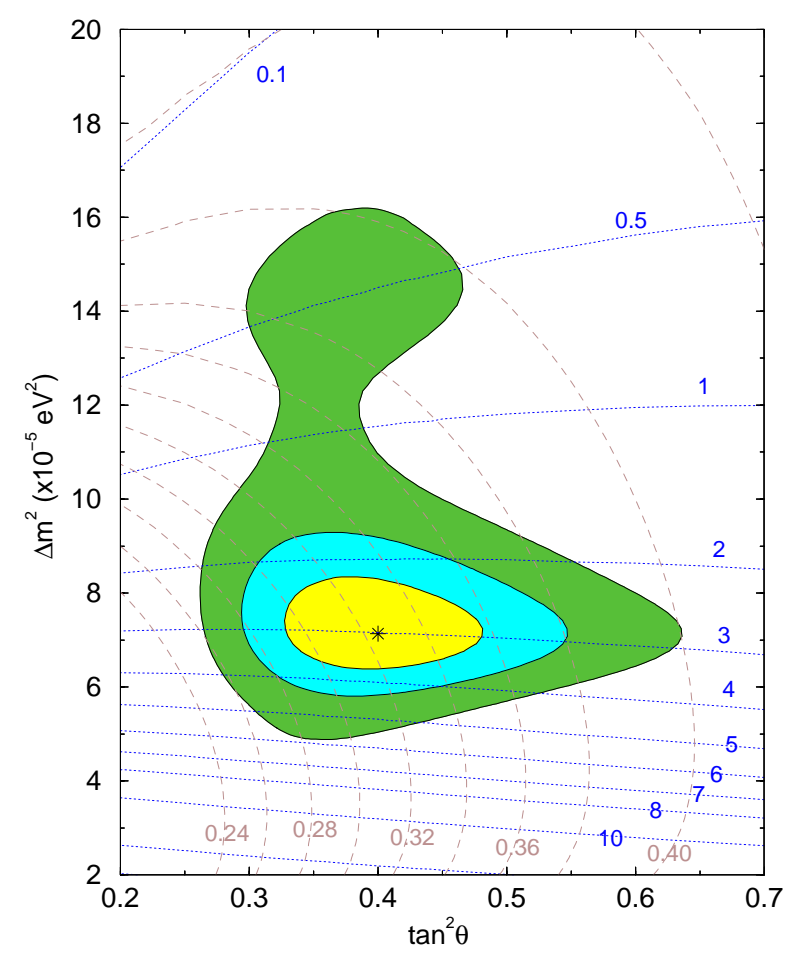

Figure 6. Predictions for the $\mathrm{CC} / \mathrm{NC}$ ratio and the Day-Night asymmetry at SNO. The dashed lines are the lines of constant CC/NC ratio (numbers at the curves) and the dotted lines show the lines of constant $A_{D N}^{S N O}$ (numbers at the curves in \%). We show also the $(1 \sigma$ and $3 \sigma)$ allowed regions of the oscillation parameters from the combined fit of the solar neutrino data and the KamLAND spectrum. The best fit point is indicated by a star.

Precise measurements of the ratio $\mathrm{CC} / \mathrm{NC}$ will also strengthen the upper bounds on mixing and $\Delta m^{2}$.

2). The day-night asymmetry at SNO. The KamLAND provides a strong lower bound on $\Delta m^{2}$, and shifts the best fit point to larger $\Delta m^{2}$. This further diminishes the expected value of day-night asymmetry. In Fig. 6 we show the contours of constant $A_{D N}^{S N O}$. The best fit point prediction and the $3 \sigma$ bound equal

$$
A_{N D}^{S N O}=3.0 \pm 0.8 \%, \quad(1 \sigma), \quad A_{N D}^{S N O}<6 \% \quad(3 \sigma) .
$$

The present best fit value of the SNO asymmetry, $7 \%$, is accepted at about $99 \%$ C.L.. The expected asymmetry at Super-Kamiokande is even smaller: In the best fit point we expect $A_{N D}^{S K} \approx(1.7-2.0) \%$.

3). The turn up of the spectrum at SNO and SuperKamiokande at low energies. Using results of [22] we predict for the best fit point (16) an increase of ratio of the observed to expected (without oscillation) number of the CC events, $R_{S N O}^{C C}$, from 0.31 at $8 \mathrm{MeV}$ to 0.345 at $5 \mathrm{MeV}$, so that

$$
\frac{R_{S N O}^{C C}(5 \mathrm{MeV})-R_{S N O}^{C C}(8 \mathrm{MeV})}{R_{S N O}^{C C}(8 \mathrm{MeV})}=0.10-0.12 .
$$

In Super-Kamiokande the turn up is about $(5-7) \%$ in the same interval (5 - 8) MeV.
4). Further KamLAND measurements. Possible impact can be estimated using Figs. 1, 4. A decrease of the error by factor of 2 (which will require both significant increase of statistics and decrease of the systematic error) will allow KamLAND alone to exclude all the regions but the l-region at 95 $\%$ C.L., if the best fit is at the same point as it is determined now.

5). BOREXINO. At $3 \sigma$ we predict the following suppressions of signals with respect to the SSM predictions to BOREXINO experiment [23], in the two allowed regions:

$$
\begin{aligned}
& R_{B}(\mathrm{l}-\text { region })=0.61-0.73, \\
& R_{B}(\mathrm{~h}-\text { region })=0.62-0.73 .
\end{aligned}
$$

So, BOREXINO will perform consistency check but it will not distinguish the $1-$ and $\mathrm{h}$ - regions.

6). Gallium production rate. In the best fit point one predicts the germanium production rate $Q_{G e}=71$ SNU. In the h-region best fit point $Q_{G e}=72 \mathrm{SNU}$. So, the Gallium experiments do not discriminate among the 1 - and $\mathrm{h}$ - regions. However, precise measurements of $Q_{G e}$ are important for improvements of the bound on the 1-2 mixing and its deviation from maximal value.

\section{Conclusions}

1. The first KamLAND results (rate and spectrum) are in a very good agreement with the predictions based on the LMA MSW solution of the solar neutrino problem.

2. Our analysis of the KamLAND data reproduces well the results of the collaboration. The oscillation parameters extracted from the KamLAND data and from the solar neutrino data agree within $1 \sigma$.

3. We have performed a combined analysis of the solar and KamLAND results. The main impact of the KamLAND results on the LMA solution can be summarized in the following way. KamLAND

- shifts the $\Delta m^{2}$ to slightly higher values $6.3 \cdot 10^{-5}$ $\rightarrow 7.1 \cdot 10^{-5} \mathrm{eV}^{2}$; (the mixing is practically unchanged: $\tan ^{2} \theta=0.40$, and this number is rather stable with respect to variations of the analysis;

- establishes rather solid lower bound on the $\Delta m^{2}$ : $\Delta m^{2}>4.8 \cdot 10^{-5} \mathrm{eV}^{2}(3 \sigma)$

4. The KamLAND results strengthen the upper bound on the expected value of the day-night asymmetry at SNO: $A_{D N}^{S N O}<6 \%$. We predict about $10 \%$ turn up of the energy spectrum at SNO in the interval of energies (5 - 8) MeV. The $\mathrm{CC} / \mathrm{NC}$ ratio is expected to be $\mathrm{CC} / \mathrm{NC} \approx 0.33$, that is, above the new SNO salt phase experimental result.

Future SNO measurements of the $\mathrm{CC} / \mathrm{NC}$ ratio and $A_{D N}^{S N O}$ will have further strong impact on the LMA parameter space.

\section{Aknowledgments}

P.C.H would like to thank FAPESP for financial support. 


\section{References}

[1] K. Eguchi et al., KamLAND collaboration, Phys. Rev. Lett. 90, 021802 (2003).

[2] J. N. Bahcall, "Neutrino astrophysics", Cambridge U. Press, Cambridge, England, 1989.

[3] B. T. Cleveland et al., Astroph. J. 496 (1998) 505; K. Lande et al., in Neutrino 2000 Sudbury, Canada 2000, Nucl. Phys. B (Proc. Suppl.) 50 (2001) 21. (Here and in ref. [5], [6], [7] we cite the latest publications of the collaboration in which the references to earlier works can be found.)

[4] K. S. Hirata et al., Phys. Rev. Lett. 651297 (1990).

[5] J. N. Abdurashitov et al. (SAGE collaboration), J. Exp. Theor. Phys. 95, 181 (2002); Zh. Eksp. Teor. Fiz. 122, 211 (2002).

[6] T. Kirsten et al., Talk given at the 20th Int. Conf on Neutrino Physics and Astriphysics, Neutrino 2002, Munich, Germany, May 25 - 30, 2002.

[7] S. Fukuda et al. (Super-Kamiokande collaboration) Phys. Rev. Lett. 86, 5651 (2001); Phys. Rev. Lett. 86, 5656 (2001).

[8] Q. R. Ahmad et al., SNO collaboration, Phys. Rev. Lett. 87, 071301 (2001).

[9] Q. R. Ahmad et al., SNO collaboration, Phys. Rev. Lett. 89, 011301 (2002).

[10] Q. R. Ahmad et al., SNO collaboration, Phys. Rev. Lett. 89, 011302 (2002).

[11] L. Wolfenstein, Phys. Rev. D17, 2369 (1978); S. P. Mikheyev and A. Yu. Smirnov, Yad. Fiz. 42, 1441 (1985) [ Sov. J. Nucl. Phys. 42, 913 (1985)], Nuovo Cim. C9, 17 (1986); S. P. Mikheyev and A. Yu. Smirnov, ZHETF, 91, (1986), [Sov. Phys. JETP, 64, 4 (1986)].
[12] V. Barger, D. Marfatia, K. Whisnant, and B.P. Wood, Phys. Lett. B537, 179 (2002); A. Bandyopadhyay, S. Choubey, S. Goswami, and D. P. Roy, Phys. Lett. B540, 14 (2002); J. N. Bahcall, M.C. Gonzalez-Garcia, and C. Peña-Garay, JHEP 0207, 054 (2002); A. Strumia, C. Cattadori, N. Ferrari, and F. Vissani, Phys. Lett. B541, 327 (2002); P. Aliani, V. Antonelli, R. Ferrari, M. Picariello, and E. Torrente-Lujan, Phys. Rev. D67, 013006 (2003); G.L. Fogli, E. Lisi, A. Marrone, D. Montanino, and A. Palazzo, Phys. Rev. D66, 053010 (2002); M. Maltoni, T. Schwetz, M.A. Tortola, and J.W.F. Valle, Phys. Rev. D67, 013011 (2003). M. Smy, hep-ex/0208004.

[13] P. C. de Holanda, A. Yu. Smirnov, Phys. Rev. D66, 113005 (2002).

[14] Q. R. Ahmad et al., SNO collaboration, Phys. Rev. Lett. 92, 181301 (2004).

[15] P. C. de Holanda, A. Yu. Smirnov, Astropart. Phys. 21, 287 (2004).

[16] J. N. Bahcall, M.H. Pinsonneault, and S. Basu, Astrophys. J. 555, 990 (2001).

[17] P. C. de Holanda, A. Yu. Smirnov, JCAP 0302, 001 (2003).

[18] L. E. Marcucci et al., Phys. Rev. C63, 015801 (2001); T.-S. Park et al., Phys. Rev. C67, 055206 (2003), and references therein.

[19] V. N. Gribov and B. Pontecorvo, Phys. Lett. B28, 493 (1969).

[20] J. N. Bahcall, M.C. Gonzalez-Garcia, C. Peña-Garay, hep$\mathrm{ph} / 0212147$.

[21] M. C. Gonzalez-Garcia, C. Peña-Garay, Y. Nir, and A. Yu. Smirnov, Phys. Rev. D63, 013007 (2001).

[22] P.I. Krastev, A.Yu. Smirnov, Phys. Rev. D65, 073022 (2002).

[23] BOREXINO Collaboration, G. Alimonti et al., Astropart. Phys. 16, 205 (2002). 